\title{
Nonperturbative renormalisation of composite operators with overlap quarks*
}

\author{
J. B. Zhanga ${ }^{\mathrm{a}}$, D. B. Leinweber ${ }^{\mathrm{a}}$, K. F. Liu ${ }^{\mathrm{b}}$, A. G. Williams ${ }^{\mathrm{a}}$ \\ ${ }^{a}$ CSSM Lattice Collaboration, \\ Special Research Center for the Subatomic Structure of Matter (CSSM) and Department of Physics, \\ University of Adelaide 5005, Australia \\ ${ }^{\mathrm{b}}$ Department of Physics and Astronomy, University of Kentucky, Lexington, KY 40506
}

We compute non-perturbatively the renormalisation constants of composite operators on a $16^{3} \times 32$ lattice with lattice spacing $a=0.093 \mathrm{fm}$ for the overlap fermion action by using the regularisation independent (RI) scheme. The quenched gauge configurations are generated by tadpole improved plaquette plus rectangle action. We test the perturbative continuum relation $Z_{A}=Z_{V}$ and $Z_{S}=Z_{P}$ and find that they agree well above $\mu=$ $1.6 \mathrm{GeV}$. We also perform a Renormalisation Group analysis at the next-to-next-to-leading order and convert the renormalisation constants to the $\overline{M S}$ scheme.

\section{INTRODUCTION}

Lattice QCD is a unique tool with which we can compute physical observables non-perturbatively from first principles. Renormalisation of lattice operators is an essential ingredient needed to deduce physical results from numerical simulations. In this paper we study the renormalisation properties of composite bilinear operators with the overlap quark action.

In principle, the renormalisation of a quark bilinear can be computed by lattice perturbation theory. However, lattice perturbation theory converges slowly and the higher-order corrections may not be small, thus introducing a large uncertainty in the calculation of the renormalised matrix elements in some continuum scheme. To overcome these difficulties, Martinelli et al. [1] have proposed a promising non-perturbative renormalisation procedure. The procedure allows a full non-perturbative computation of the matrix elements of composite operators in the Regularisation Independent (RI) scheme 112. The matching between the RI scheme and $\overline{M S}$, which is intrinsically perturbative, is computed using only continuum perturbation theory and has been carried out to higher orders, which is well behaved. This method has been shown to be

*Presented by J. B. Zhang quite successful in reproducing results obtained by other methods, such as using chiral Ward Identities (WI) 3. The method has also been successfully applied to determine renormalisation coefficients for various operators using the Wilson 4567 and staggered actions 8, domain-wall fermions [9], as well as the quark mass renormalisation constant for overlap fermions [10. The purpose of the current work is to study the application of this non-perturbative renormalisation procedure to the renormalisation of the quark field and the flavour non-singlet fermion bilinear operators in the case of overlap fermions.

We shall use Neuberger's overlap fermions 11 which have lattice chiral symmetry at finite cutoff. As a result, many chiral-symmetry relations are still valid 1213 and the quark propagator 14] preserves the same structure as in the continuum. The use of the overlap action entails many theoretical advantages 1516, such as no additive mass renormalisation, no $\mathcal{O}(a)$ error, and no mixing among operators of different chirality. The latter is very helpful for computing weak matrix elements.

\section{NON-PERTURBATIVE RENOR- MALIZATION METHOD}

In this section we review the nonperturbative renormalisation method of Ref. [1, which 
we use to compute the renormalisation constants of quark bilinears in this paper. The method imposes renormalisation conditions nonperturbatively, directly on quark and gluon Green's functions, in a fixed gauge, for example the Landau gauge.

Let $S(x, 0)$ denotes the quark propagator on a gauge-fixed configuration from a source 0 to all space-time points $x$. The momentum space propagator is defined as the discrete Fourier transform over the sink positions

$S(p, 0)=\sum_{x} \exp (-i p \cdot x) S(x, 0)$.

We use periodic boundary conditions in spatial directions and an anti-periodic boundary condition in time direction. The dimensionless lattice momenta are

$p_{i}=\frac{2 \pi}{N_{s}}\left(n_{i}-\frac{N_{S}}{2}\right), \quad p_{t}=\frac{2 \pi}{N_{t}}\left(n_{t}-\frac{1}{2}-\frac{N_{t}}{2}\right)$

for an $N_{s}^{3} \times N_{t}$ lattice, where $n_{i}\left(n_{t}\right)$ may in principle lie in the range $0 \rightarrow N_{s}\left(N_{t}\right)$. In practice, however, only a subset of this range is used. In this paper the momentum range was restricted to those momenta for which $n_{i}=0,1,2$ and $n_{t}=1,2,3,4$.

We also define the square of the absolute momentum,

$$
(p a)^{2}=a^{2} \sum_{\mu=0}^{3} p_{\mu} p_{\mu} .
$$

\subsection{Three Point Function}

Consider the two-fermion operators

$O_{\Gamma}(x)=\bar{\psi}(x) \Gamma \psi$,

where $\Gamma$ is the Dirac gamma matrix

$\Gamma \in\left\{1, \gamma_{\mu}, \gamma_{5}, \gamma_{\mu} \gamma_{5}, \sigma_{\mu \nu}\right\}$,

and the corresponding notation will be $\{\mathrm{S}, \mathrm{V}, \mathrm{P}$, $\mathrm{A}, \mathrm{T}\}$ respectively. The three point function with the operator insertion at position 0 and the propagator from $y$ to 0 and then from 0 to $x$ is given by

$$
\begin{aligned}
G_{O}(x, 0, y) & =\left\langle\psi(x) O_{\Gamma}(0) \bar{\psi}(y)\right\rangle \\
& =\langle S(x, 0) \Gamma S(0, y)\rangle,
\end{aligned}
$$

where $S(0, y)$ is the quark propagator from $y$ to 0 . It is the inverse of the Dirac operator.

The Fourier transform of the three-point function is given by

$$
\begin{aligned}
G_{O}(p a, q a) & \equiv \int d^{4} x d^{4} y e^{-i(p \cdot x-q \cdot y)} G_{O}(x, 0, y) \\
& =\left\langle S(p, 0) \Gamma\left(\gamma_{5} S^{\dagger}(q, 0) \gamma_{5}\right)\right\rangle . \quad(7)
\end{aligned}
$$

From this, one can define the amputated threepoint function

$\Lambda_{O}(p a, q a)=S(p a)^{-1} G_{O}(p a, q a) S(q a)^{-1}$,

where

$S(p a)=\langle S(p, 0)\rangle$,

which is translational invariant and a $12 \times 12$ matrix.

Finally, we define a projected vertex function

$\Gamma_{O}(p a)=\frac{1}{\operatorname{Tr}\left(\hat{P}_{O}^{2}\right)} \operatorname{Tr}\left(\Lambda_{O}(p a, p a) \hat{P}_{O}\right)$,

where $\hat{P}_{O}=\Gamma$ is the corresponding projection operator.

\subsection{RI/MOM Renormalisation Condition}

The renormalised operator $O(\mu)$ at scale $\mu$ is related to the bare operator

$O(\mu)=Z_{O}(\mu a, g(a)) O(a)$,

and the renormalisation condition is imposed on the vertex function $\Gamma_{O}(p a)$ at a scale $p^{2}=\mu^{2}$,

$\left.\Gamma_{O, \text { ren }}(p a)\right|_{p^{2}=\mu^{2}}=\left.\frac{Z_{O}}{Z_{\psi}} \Gamma_{O}(p a)\right|_{p^{2}=\mu^{2}}=1$,

to make it agree with the tree-level value of unity. Here $Z_{\psi}$ is the field or wavefunction renormalisation

$\psi_{\text {ren }}=Z_{\psi}^{1 / 2} \psi$.

In order to obtain the renormalisation constant $Z_{O}$ for the operator $O$, one needs to know $Z_{\psi}$ first. It has been suggested that $Z_{\psi}$ be obtained from the renormalisation of vector or axial-vector currents. For example, if one uses the conserved vector current, then one expects $Z_{V^{C}}=1$. Therefore, from the renormalisation condition Eq. (12), one obtains,

$Z_{\psi}=\left.\frac{1}{48} \operatorname{Tr}\left(\Lambda_{V_{\mu}^{C}}(p a) \gamma_{\mu}\right)\right|_{p^{2}=\mu^{2}}$. 
However, in this work, we will obtain $Z_{\psi}$ directly from the quark propagator. It can be defined from the Ward Identity (WI) as [1]

$Z_{\psi}=-\left.i \frac{1}{12} \operatorname{Tr}\left[\frac{\partial S(p a)^{-1}}{\not p p}\right]\right|_{p^{2}=\mu^{2}}$.

To avoid derivatives with respect to a discrete variable, we have used

$Z_{\psi}^{\prime}=-\left.i \frac{1}{12} \frac{\operatorname{Tr} \sum_{\mu=1,4} \gamma_{\mu}\left(p_{\mu} a\right) S(p a)^{-1}}{4 \sum_{\mu=1,4}\left(p_{\mu} a\right)^{2}}\right|_{p^{2}=\mu^{2}}$

which, in the Landau Gauge, differs from $Z_{\psi}$ by a finite term of order $\alpha_{s}^{2}$ [17, and the difference is less than $1 \%$ at the typical scale $\mu \sim 2 \mathrm{GeV}$. It has been pointed out in Ref. 9 that the systematic error due to the definition of lattice momentum $p$ is much larger than $1 \%$. We will not distinguish $Z_{\psi}^{\prime}$ and $Z_{\psi}$ later on.

\section{NUMERICAL RESULTS}

We work on a $16^{3} \times 32$ lattice with lattice spacing, $a=0.093 \mathrm{fm}$. The gauge configurations are created using a tadpole improved plaquette plus rectangle (Lüscher-Weisz [18]) gauge action through the pseudo-heat-bath algorithm. A total of 50 configurations are considered. The lattice parameters are summarised in Table 1 . The lattice spacing is determined from the static quark potential with a string tension $\sqrt{\sigma}=$ $440 \mathrm{MeV}[19$.

Table 1

Lattice parameters.

\begin{tabular}{ccccc}
\hline Action & Volume & $N_{\text {Samp }}$ & $\beta$ & $a(\mathrm{fm})$ \\
\hline Improved & $16^{3} \times 32$ & 500 & 4.80 & 0.093 \\
\hline
\end{tabular}

The gauge field configurations are gauge fixed to the Landau gauge using a Conjugate Gradient Fourier Acceleration 20] algorithm with an accuracy of $\theta \equiv \sum\left|\partial_{\mu} A_{\mu}(x)\right|^{2}<10^{-12}$. We use an improved gauge-fixing scheme 21] to minimise gauge-fixing discretisation errors.

The massive overlap Dirac operator [1] is defined so that at tree-level there is no mass or wavefunction renormalisation [22,

$D\left(m_{0}\right)=\rho+\frac{m_{0}}{2}+\left(\rho-\frac{m_{0}}{2}\right) \gamma_{5} \epsilon(H)$.
Here $\epsilon(H)=H / \sqrt{H^{2}}$ is the matrix sign function and $H$ is taken to be the hermitian WilsonDirac operator, i.e., $H=\gamma_{5} D_{\mathrm{W}}$. Here $D_{\mathrm{W}}$ is the usual Wilson fermion operator, but with a negative mass parameter $-\rho=1 / 2 \kappa-4$ in which $\kappa_{c}<\kappa<0.25$.

Our numerical calculation begins with an evaluation of the inverse of $D\left(m_{0}\right)$ for each gauge configuration in the ensemble. We use a 14th order Zolotarev approximation 23] to the sign matrix function $\epsilon\left(H_{w}\right)$, and in the selected window of $x \in[0.031,2.5]$ of $\epsilon(x)$, the approximation is better than $3.3 \times 10^{-10}$ 24]. In the calculations, $\kappa=0.19163$ is used, which gives $\rho a=1.391$. We calculate 15 quark masses by using a shifted version of a Conjugate Gradient solver. The bare quark masses $m_{0} a$ are chosen to be 0.0250 , $0.0278,0.0334,0.0389,0.0445,0.0501,0.0584$, $0.0668,0.0834,0.1001,0.1252,0.1669,0.2086$, $0.2503,0.2921$. The $m_{0}$ values in physical units are $53,59,71,83,94,106,124,142,177,212$, $266,354,442,531$, and $620 \mathrm{MeV}$ respectively.

\subsection{Axial and vector currents}

Let us consider first the vector and axial vector currents. Since each obeys a chiral WI 25] their renormalisation constants are finite. In Fig. 1] we show $\Gamma_{A}$ and $\Gamma_{V}$, calculated in the RI scheme for different bare quark masses as a function of the lattice momentum $(p a)^{2}$. We find they are weakly dependent on the mass, and almost scale independent for $(p a)^{2} \geq 0.5$.

For the overlap fermion, which satisfies the Ginsparg-Wilson [26] relation and preserves chiral symmetry on finite lattice. In Ref. 27] it has been proved that the renormalisation constants for naive vector and axial vector currents satisfy

$Z_{A}=Z_{V}$

In Fig. 2 we show the quantities $\Gamma_{A}-\Gamma_{V}$ and and $\frac{1}{2}\left(\Gamma_{A}+\Gamma_{V}\right)$, after extrapolating to the chiral limit. We can see from upper part of the figure that there is no signal of effects from chiral symmetry breaking, since $\Gamma_{A}-\Gamma_{V}$ is tending to zero at moderate and high momenta. The effects of spontaneous chiral symmetry breaking are visible at low momenta and they are damped at higher momenta. At the momenta of interest, there also 

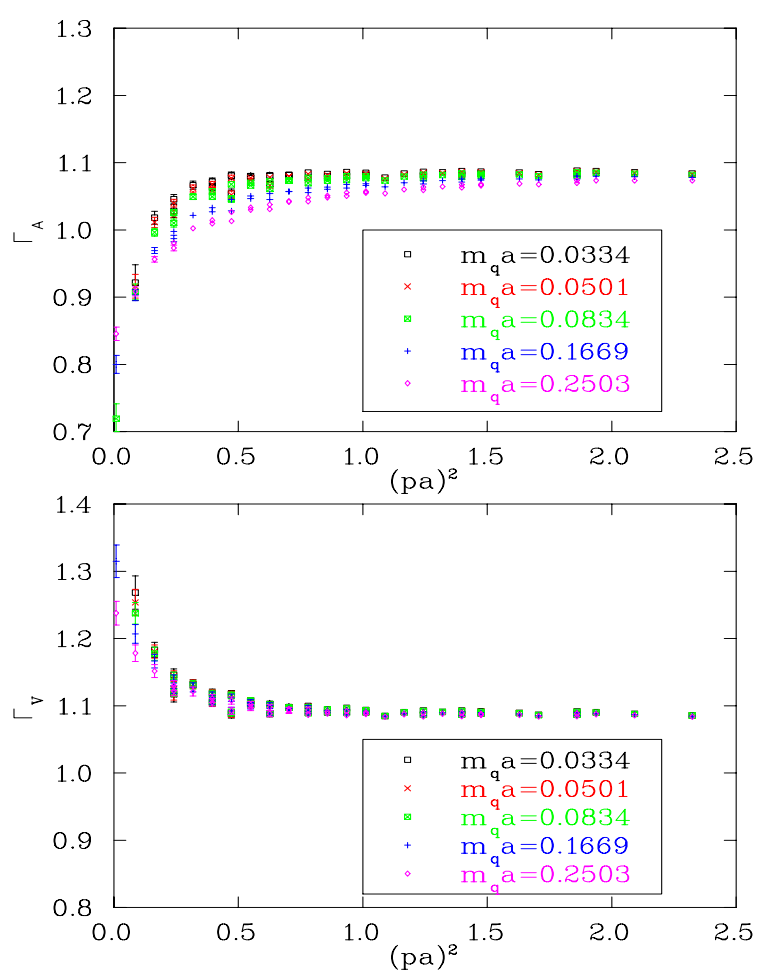

Figure 1. The projected vertex function $\Gamma$ defined in Eq. (10) for vector and axial vector currents with different bare quark masses. By the renormalisation condition (Eq.(12) ), they are the ratio of renormalisation constant $Z_{\psi} / Z_{A}$ and $Z_{\psi} / Z_{V}$ respectively.

seems to be no significant splitting due to nonperturbative effects with the difference between $\Gamma_{A}$ and $\Gamma_{V}$ being less than $1 \%$ at $(a p)^{2}=0.7$ and smaller for momenta above this. In the lower part of Fig. 2 we plot $\frac{1}{2}\left(\Gamma_{A}+\Gamma_{V}\right)$ against $(a p)^{2}$, and it can be used for the extraction of both $Z_{A} / Z_{\psi}$ and $Z_{V} / Z_{\psi}$ to increase the statistical accuracy.

\subsection{Pseudoscalar and scalar densities}

The pseudoscalar and scalar densities differ from the axial and vector currents in that their renormalisation is not scale independent. For overlap fermions with chiral symmetry the RI scheme preserves the well known $\overline{M S}$ relations

$Z_{S}=Z_{P} \quad$ and $\quad Z_{m}=\frac{1}{Z_{S}}$

and the quantities $Z_{S} / Z_{P}, Z_{S} Z_{m}$ and $Z_{m} Z_{P}$ are expected to be scale independent.
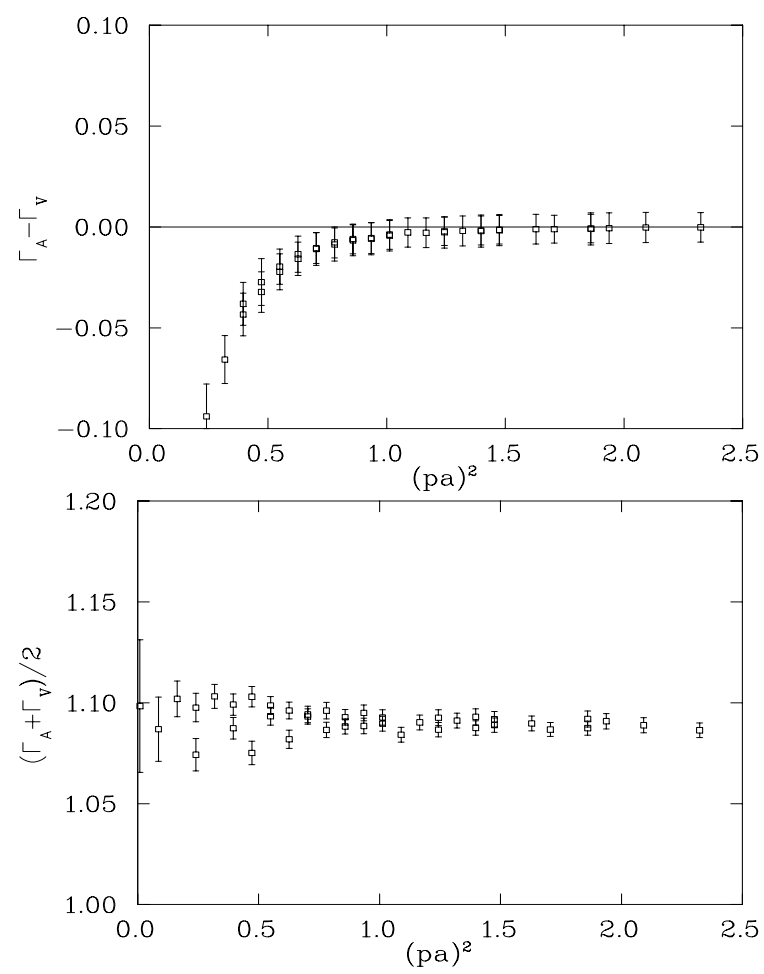

Figure 2. Checking $Z_{A}=Z_{V}$, the upper plot is $\left(\Gamma_{A}-\Gamma_{V}\right)$ versus $(p a)^{2}$, where we linear extrapolate to the chiral limit $\left(m_{0}=0\right)$. The lower plot is $\frac{1}{2}$ $\left(\Gamma_{A}+\Gamma_{V}\right)$ versus $(p a)^{2}$. The relation $Z_{A}=Z_{V}$ is valid at moderate to large $(p a)^{2}$.

Fig. 3 and Fig. 4 show $\Gamma_{S}$ and $\Gamma_{P}$ versus $(p a)^{2}$ with different masses. Unlike in the case of the vector and axial vector currents, they are strongly dependent on the quark mass $m_{0}$ for light quarks. When we want to extract the chiral limit value, we cannot use a simple linear or quadratic fit. In Fig. 3] and Fig. 4 we can clearly see the pole effect in the small $(p a)^{2}$ region.

As discussed in detail in Ref. 9, due to the contribution from the zero-modes, we may fit the $\Gamma_{S}$ to the form

$\Gamma_{S}=\frac{c_{1, S}}{\left(a m_{0}\right)^{2}}+c_{2, S}+c_{3, S}\left(a m_{0}\right)^{2}$

at a fixed momentum with $Z_{\psi} / Z_{S}$ being given by $c_{2, S}$. The fitted value of $c_{2, S}$ is plotted in Fig. [5] as $Z_{\psi} / Z_{S}$. We find that the coefficient of the pole term, $c_{1, S}$ is very small, between $10^{-3}$ to $10^{-4}$, this means that the pole term only provides a 


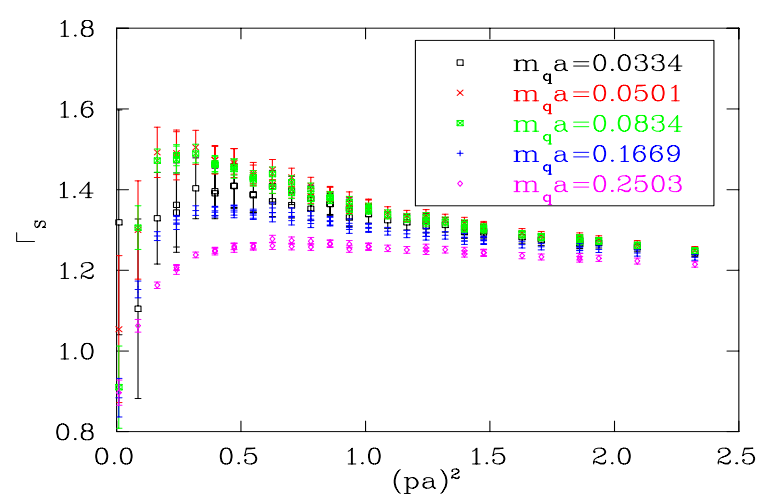

Figure $3 . \Gamma_{S}$ versus $(p a)^{2}$ with different masses. Here one can see the strong mass dependence and nonmonotonic behaviour.

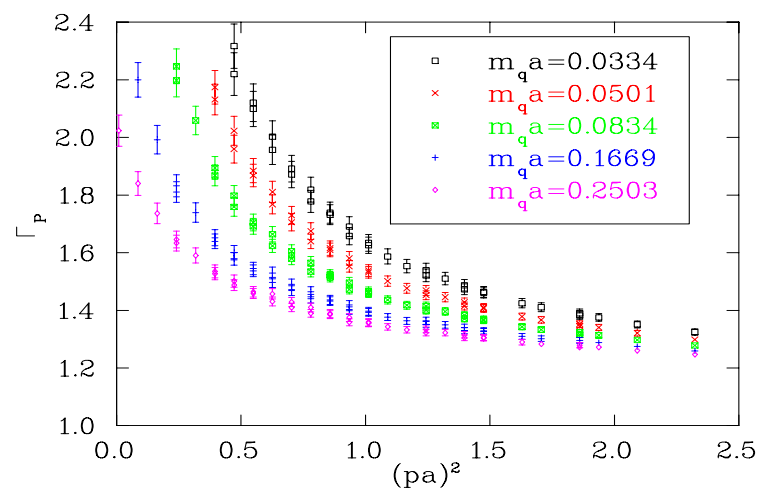

Figure 4. $\Gamma_{P}$ versus $(p a)^{2}$ with different masses. Here also we have strong mass dependence and pole behaviour.

large contribution at very small $m_{0}$, i.e., near the chiral limit.

For $\Gamma_{P}$, we fit to the form

$\Gamma_{P, \text { latt }}=\frac{c_{1, P}}{\left(a m_{0}\right)^{2}}+\frac{c_{2, P}}{\left(a m_{0}\right)}+c_{3, P}+c_{4, P}\left(a m_{0}\right)^{2}$,

with $c_{3, P}$ being equal to $Z_{\psi} / Z_{P}$. The quadratic mass pole is due to zero-mode effects in $\langle\bar{q} q\rangle$. The fitted value of $c_{3, P}$ is plotted in Fig. [5] as $Z_{\psi} / Z_{P}$.

As the evidence for the above fitting form, we consider the resulting values for $Z_{\psi} / Z_{S}$ and $Z_{\psi} / Z_{P}$. Fig. 5 shows a comparison between the extracted values of these two quantities. As chiral symmetry would predict for $Z_{\psi} / Z_{S}$ and $Z_{\psi} / Z_{P}$, the two quantities coincide at moderate and large momenta.This provides an excellent test of both the fitting method to extract the poles and the

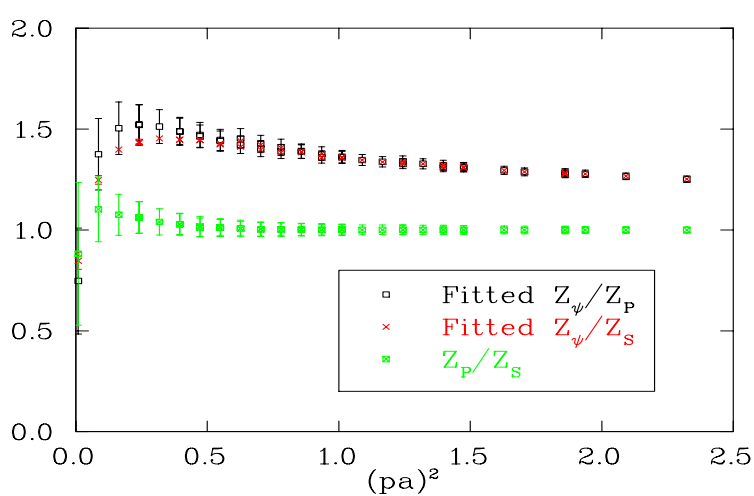

Figure 5. Checking of $Z_{P}=Z_{S}$ after mass pole subtraction.

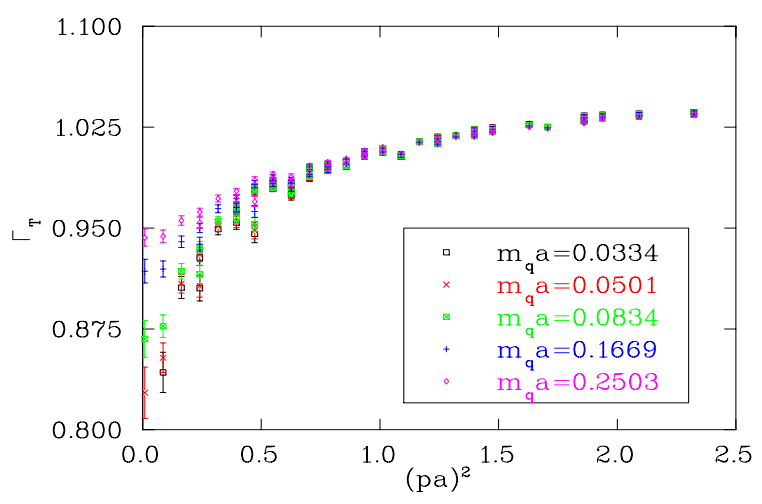

Figure 6. $\Gamma_{T}$ versus $(p a)^{2}$ with different masses. It shows no significant mass dependence for moderate to large $(p a)^{2}$.

chiral properties of overlap fermions.

\subsection{The Tensor current}

In Fig. 6] we show $\Gamma_{T}$ versus $(p a)^{2}$ with different masses. We can see that at moderate and large $(p a)^{2}, \Gamma_{T}$ is not sensitive to the quark masses. The chiral limit value is obtained by a linear fit in quark mass as for the cases of vector and axial vector currents, and the results will be presented in the next subsection.

\subsection{Running of the renormalisation con- stants}

The renormalised operators are defined as

$Z_{O} O_{\text {bare }}=O_{\text {ren }}$.

Requiring the bare operator to be independent of the renormalisation scale $\mu^{2}$ gives the RG equa- 


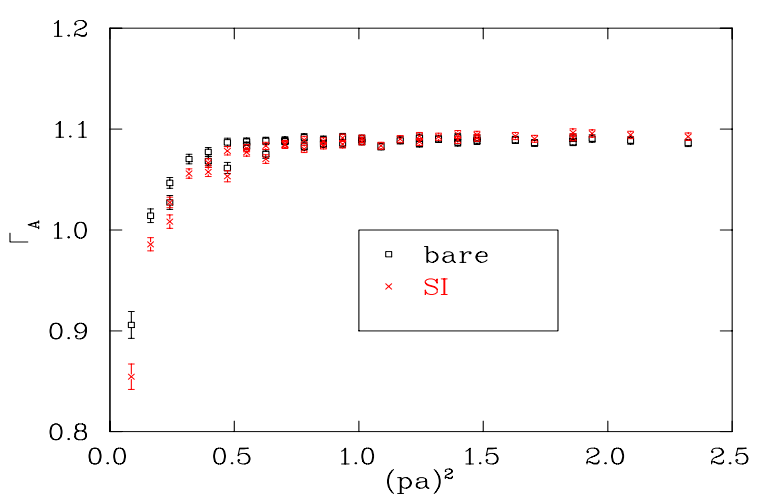

Figure 7. $\Gamma_{A}$ versus $(p a)^{2}$ in the chiral limit before (labelled as "bare") and after (labelled as "SI") three loop perturbative running, such that they coincide at $(p a)^{2}=1$. The latter is almost $(p a)^{2}$ independent after $(p a)^{2}>0.6$. The slope versus $(p a)^{2}$ is about 0.01 and is interpreted as an $O\left(a^{2}\right)$ effect.

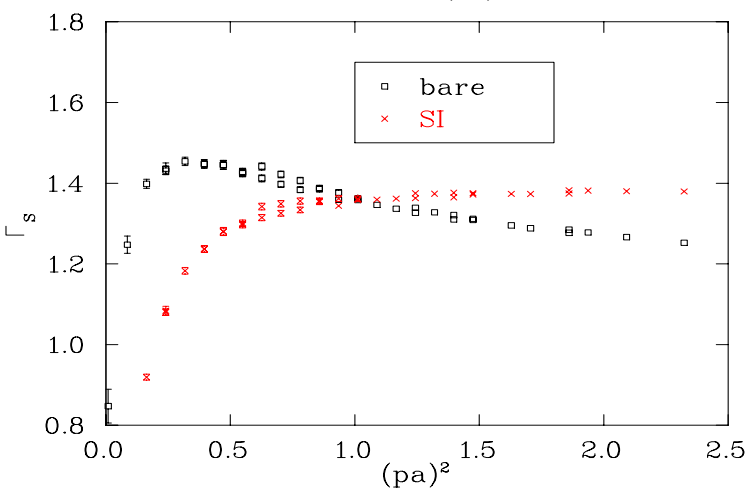

Figure 8. The same as Fig. (7). Here is $\Gamma_{S}$ versus $(p a)^{2}$. The slope of SI versus $(p a)^{2}$ is about 0.02 .

tion,

$\mu^{2} \frac{d}{d \mu^{2}} O_{\text {ren }}=\frac{1}{Z_{O}} \mu^{2} \frac{d Z_{O}}{d \mu^{2}} O_{\text {ren }}=-\frac{\gamma_{O}}{2} O_{\text {ren }}$.

The solution of $Z_{O}\left(\mu^{2}\right)$ can be written in the following form

$Z_{O}\left(\mu^{2}\right)=\frac{C_{O}\left(\mu^{2}\right)}{C_{O}\left(\mu^{\prime 2}\right)} Z_{O}\left(\mu^{\prime 2}\right)$.

The coefficient $C_{O}\left(\mu^{2}\right)$ can be found in Appendix B of Ref. 9] and references within.

In this work, the value of $\alpha_{s}$ was calculated at three loops using a lattice value of $\Lambda_{\mathrm{QCD}}$ taken from Ref. 28] as $\Lambda_{\mathrm{QCD}}=238 \pm 19 \mathrm{MeV}$.

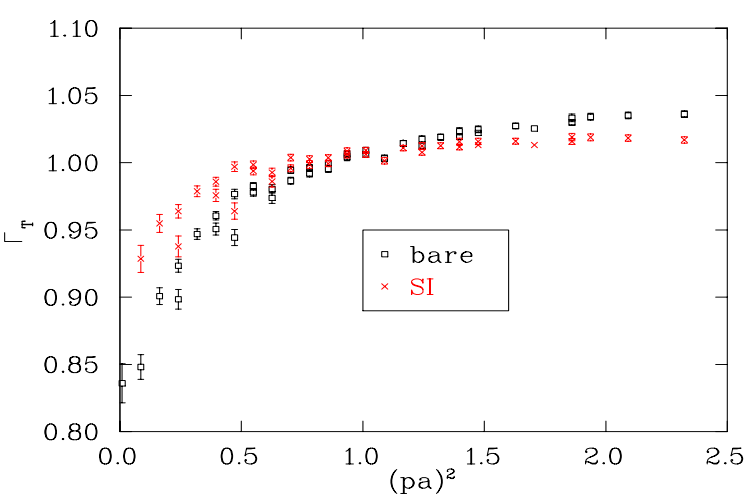

Figure 9. $\Gamma_{T}$ versus $(p a)^{2}$ in the chiral limit before and after two loop perturbative running. The latter is almost $(p a)^{2}$ independent after $(p a)^{2}>0.6$, the slope versus $(p a)^{2}$ is about 0.01 .

Both $Z_{A}$ and $Z_{V}$ should be scale independent, but this is not the case for $Z_{\psi}$. Fig. 7 shows both $\Gamma_{A}$ and the scale invariant (SI) quantity (the data after removing the renormalisation group running) calculated as described above:

$\Gamma_{A}^{S I}\left((a p)^{2}\right)=\Gamma_{A}\left((a p)^{2}\right) / C_{\psi}\left((a p)^{2}\right)$.

The quantity $C_{\psi}$ is normalised so that $C_{\psi}(1)=1$. As can be seen, in this case the renormalisation group running is coming from $Z_{\psi}$ alone. It is small, but it actually improves the scale independence of the data. The remain scale dependence of this data is very small and a plausible explanation for this is a small $(a p)^{2}$ error. Indeed, when a linear fit of the SI data versus $(a p)^{2}$ is performed, for $0.8<(a p)^{2}<2.0$, the gradient is $\approx 0.01$.

In the case of $Z_{\psi} / Z_{S}$, the two renormalisation constants are both running with $(a p)^{2}$. Fig. 8 shows both $\Gamma_{S}$ and the scale invariant (SI) quantity after three loop running. The modification to the raw data, which is taken after the mass-pole has been subtracted gives a satisfactory result. The linear fit of the SI data versus $(a p)^{2}$ in the range of $0.8<(a p)^{2}<2.3$, gives a gradient of about 0.02 .

The SI result of $\Gamma_{T}$ which comes from the two loop running of $Z_{T}$ [29] and three loop running of $Z_{\psi}$ is plotted in Fig. 9] The "bare" data is taken by a simple linear extrapolation to the chiral limit. The linear fit to the SI data gives gradient about 0.01 . 


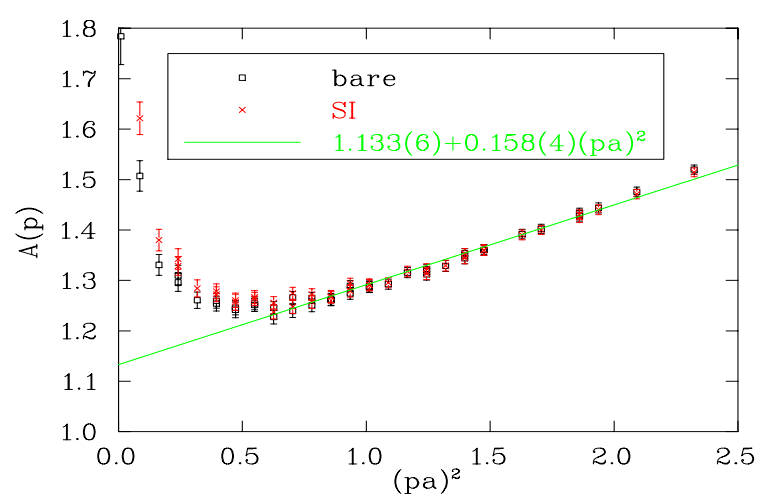

Figure 10. $A(p)$ in the chiral limit and its scale invariant counterpart versus $(p a)^{2}$ as $a+b(p a)^{2}$. The fitting range is $[0.70,2.32]$, which gives $Z_{\psi}$ about $1.133 \pm 0.006$.

With the interpretation that the remaining scale dependence is due to $\mathcal{O}(a p)^{2}$ effects, the correct way to extract the renormalisation coefficients is to first construct the SI quantity as described above, and then fit any remaining scale dependence 4 to the form

$y=c_{1}+c_{2}(a p)^{2}$,

for a range of momenta that is chosen to be "above" the region for which condensate effects are important. We choose the range $0.8<$ $(a p)^{2}<2.3$. We then apply the renormalisation group running formula (an inverse operation of Eq. (25) ) to $c_{1}$ in Eq. (26) to get the ratio of the renormalisation constant which is now free of $(a p)^{2}$ error in RI scheme.

\subsection{Extracting $Z_{\psi}$ from the propagator}

Here we will use Eq. (16) to extract the wave function renormalisation constant $Z_{\psi}$. We will use two different definitions of lattice momenta, i.e. , the discrete lattice momentum defined in Eq. (2) and the kinematic lattice momentum $q_{\mu}$ which was introduced in Eq. (A9) of Ref. [30].

\subsubsection{Discrete lattice momentum $p_{\mu}$}

We here use the discrete lattice momentum $p_{\mu}$ in Eq. (16) to calculate $Z_{\psi}^{\prime}$. By using Eq. (16), we can get the corresponding quantity of $Z_{\psi}^{\prime}$ (here we use the symbol $A(p)$ for $\left.Z_{\psi}^{\prime}\right)$. The resulting $A(p)$ is strongly dependent on the scale. We use three loop perturbative running in RI scheme and fi-

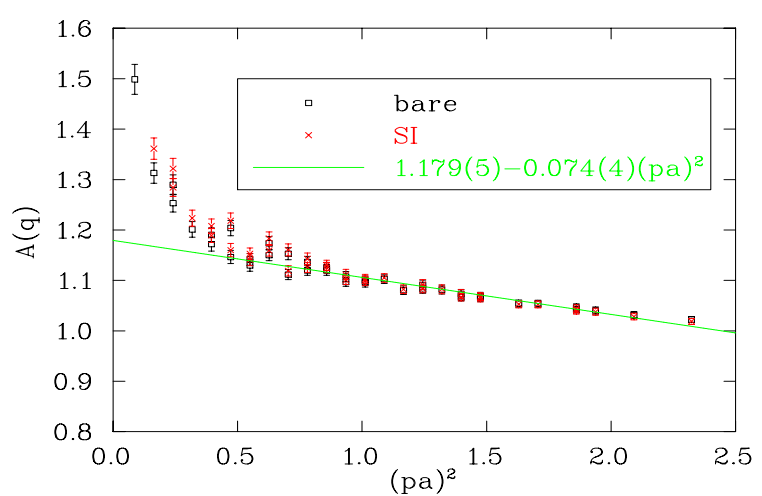

Figure 11. $Z_{\psi}$ calculated from Eq. (15) by using the kinematic lattice momentum $q_{\mu}$ instead of the discrete lattice momentum $p_{\mu}$.

nally extract the value of $Z_{\psi}$ from $A(p)$ by fitting $A(p)$ to Eq. (26) for a range of momenta for which the condensate effects are unimportant. Here we chose the range $(a p)^{2} \subset(0.70,2.32)$, which corresponds to $p \in(1.78,3.23) \mathrm{GeV} . c_{1}$ can be identified as the SI value of $Z_{\psi}$. In Fig. 10 we show a linear fit for $A(p)$ versus $(p a)^{2}$ as in Eq. (26).

\subsubsection{Kinematic lattice momentum $q_{\mu}$}

Another way to calculate $Z_{\psi}$ is to use the kinematic lattice momentum $q_{\mu}$ defined in Eq. (A9) of Ref. 30. The calculations are similar to those using $p_{\mu}$. The result is plotted in Fig. [1] The fitted value of $Z_{\psi}$ is $1.179(5)$, with the difference to the value calculated by $p_{\mu}$ being less than $4 \%$. In both calculations, we attribute the remaining $(p a)^{2}$ dependence to the possible $\mathcal{O}\left((p a)^{2}\right)$ error and use the linear fit Eq. (26) to remove it. When using the kinematic lattice momentum $q_{\mu}$ to calculate $Z_{\psi}$, the $\mathcal{O}\left((p a)^{2}\right)$ errors are much smaller than in the case of using the discrete lattice momentum $p_{\mu}$.

\subsection{Matching to $\overline{M S}$ scheme}

In order to confront experiment, one frequently prefers to quote the final results in the $\overline{M S}$ scheme at a certain scale. For light hadrons, the popular scale is $2 \mathrm{GeV}$.

The perturbative expansion of the ratio $Z^{\overline{M S}} / Z^{R I}$ to two-loop order is given by $[4$

$R=\frac{Z^{\overline{M S}}}{Z^{R I}}=1+\frac{\alpha_{s}}{4 \pi}\left(Z^{R I}\right)_{0}^{(1)}+\left(\frac{\alpha_{s}}{4 \pi}\right)^{2}\left(Z^{R I}\right)_{0}^{(2)}+\ldots$ 
The numerical values of the matching coefficients, $Z_{0}^{(1)}$ and $Z_{0}^{(2)}$ in Eq. (27) for $Z_{\psi}$ and $Z_{S}$ can be found in Appendix $C$ of Ref. 9, we have not found the numerical value for $Z_{T}$ in the literature. The final results for the renormalisation constants are listed in Table 2

Table 2

Final Z-factor results

\begin{tabular}{ccc}
\hline$Z-$ factor & $\mathrm{RI}(2 \mathrm{GeV})$ & $\overline{M S}$ at $2 \mathrm{GeV}$ \\
\hline$Z_{A} / Z_{\psi}$ & $0.924 \pm 0.004$ & $0.928 \pm 0.004$ \\
$Z_{S} / Z_{\psi}$ & $0.739 \pm 0.003$ & $0.842 \pm 0.004$ \\
$Z_{T} / Z_{\psi}$ & $1.009 \pm 0.002$ & $1.012 \pm 0.002$ \\
$Z_{\psi}($ from $p)$ & $1.134 \pm 0.006$ & $1.130 \pm 0.006$ \\
$Z_{\psi}($ from $q)$ & $1.180 \pm 0.005$ & $1.175 \pm 0.005$ \\
\hline
\end{tabular}

\section{SUMMARY}

In this work we performed the non-perturbative renormalisation of composite operators with overlap fermions. The main results are: in $\overline{M S}$ scheme at $\mu=2 \mathrm{GeV}, Z_{\psi}=1.153(5)(22), Z_{S}$ $=0.970(4)(20), Z_{A}=1.070(5)(21)$, and $Z_{T}=$ $1.167(3)(23)$, where the first error is statistical and the second error comes from using the two different lattice momenta to get $Z_{\psi}$.

Support for this research from the Australian Research Council is gratefully acknowledged.

\section{REFERENCES}

1. G. Martinelli, C. Pittori, C.T. Sachrajda, M. Testa and A. Vladikas, Nucl. Phys. B 445, 81 (1995).

2. M. Ciuchini, E. Franco, G. Martinelli, L. Reina, L. Silvestrini, Z. Phys. C 68, 239 (1995).

3. G. Martinelli, S. Petrarca, C.T. Sachrajda and A. Vladikas, Phys. Lett. B 311,241 (1993), E: B317, 660 (1993).

4. V. Gimenez, L. Giusti, F. Rapuano, M. Talevi, Nucl. Phys. B531, 429 (1998).

5. A. Donini, V. Gimenez, G. Martinelli, M. Talevi and A. Vladikas, Eur. Phys. J. C 10, 121 (1999).

6. L. Giusti et al., Nucl. Phys. (Proc. Suppl.) B 73, 210 (1999).
7. D. Becirevic et al., Phys. Lett. B 444, 401 (1998).

8. JLQCD Collaboration, S. Aoki et al., Nucl. Phys. (Proc.Suppl.) B 73, 279 (1999).

9. T. Blum et al., Phys. Rev. D 66, 014504 (2002).

10. L. Giusti, C. Hoelbling, C. Rebbi, Phys. Rev. D, 64, 114508 (2001). Erratum-ibid. D, 65, 079903,(2002).

11. H. Neuberger, Phys. Lett. B417, 141 (1998), Phys. Lett. B427 353 (1998).

12. R. Narayanan, H. Neuberger, Phys. Lett. B302, 62 (1993), Nucl. Phys. B 443, 305 (1995).

13. M. Lüscher, Phys. Lett. B428, 342 (1998).

14. K.F. Liu, hep-lat/0206002

15. H. Neuberger, Nucl. Phys. (Proc.Suppl.) B 83, 67 (2000).

16. S. J. Dong, F. X. Lee, K. F. Liu, and J. B. Zhang, Phys. Rev. Lett. 85, 5051 (2000).

17. E. Franco and V. Lubicz, Nucl. Phys. B531, 641 (1998)

18. M. Lüscher and P. Weisz, Commun. Math. Phys. 97, 59 (1985).

19. F. D. R. Bonnet et al., hep-lat/9912044.

20. A. Cucchieri and T. Mendes, Phys. Rev. D 57, 3822 (1998).

21. F. D. R. Bonnet et al., Austral J. Phys. 52, 939 (1999).

22. S. J. Dong, T. Draper, I. Horváth, F. X. Lee, K. F. Liu, and J. B. Zhang, Phys. Rev. D 65, 054507 (2002).

23. J. van den Eshof et al., Comput. Phys. Commun. 146, 203 (2002); Nucl. Phys. (Proc. Suppl.) 106, 1070(2002).

24. S. J. Dong et al., hep-lat/0304005

25. M. Bochicchio, L. Maiani, G. Martinelli, G.C. Rossi and M. Testa, Nucl. Phys. B 262, 331 (1985).

26. P. H. Ginsparg, K. G. Wilson, Phys. Rev. D, 25, 2649 (1982).

27. C. Alexandrou, E. Follana, H. Panagopoulos, E. Vicari, Nucl. Phys. B 580, 394 (2000).

28. S. Capitani, M.Luscher, R. Sommer and H. Wittig, Nucl. Phys. B 544, 669 (1999),

29. J. A. Gracey, Nucl. Phys. B 667, 242 (2003).

30. F. D. R. Bonnet et al., Phys. Rev. D 65, 114503 (2002). 